\title{
An Analysis of the Finite Element Method Using Lagrange Multipliers for the Stationary Stokes Equations
}

\author{
By Richard S. Falk*
}

\begin{abstract}
An error analysis is presented for the approximation of the stationary Stokes equations by a finite element method using Lagrange multipliers.
\end{abstract}

1. Introduction. The purpose of this note is to examine a finite element method using Lagrange multipliers for the stationary Stokes equations. Such a method is mentioned in [4] although specific details of the analysis are not provided. In this paper we shall present an analysis from a different viewpoint and then obtain error estimates under different hypotheses than considered in [4]. A similar approach has been used in [9] to analyze a finite element method using Lagrange multipliers for a second order elliptic boundary value problem with Dirichlet type boundary conditions. Some other finite element methods for the problem we consider here can be found in [5] , [6], [7] , [8] and [13].

We will consider then the approximation of

Problem (P): Find $\vec{u}=\left(u_{1}, \ldots, u_{N}\right)$ and $p$ defined on $\Omega$ such that

$$
\begin{aligned}
-v \Delta \vec{u}+\overrightarrow{\operatorname{grad} p}=\vec{f} & \text { in } \Omega, \\
\operatorname{div} \vec{u}=0 & \text { in } \Omega, \\
\vec{u}=\overrightarrow{0} & \text { on } \partial \Omega,
\end{aligned}
$$

where $\vec{u}$ is the fluid velocity, $p$ is the pressure, $\vec{f}$ are the body forces per unit mass, and $\nu>0$ is the dynamic viscosity.

The approach we will take is to consider Problem (P) in the following form. Let $a(\vec{u}, \vec{v})$ denote the bilinear form

$$
\int_{\Omega} \nu \sum_{i=1}^{N} \frac{\partial \vec{u}}{\partial x_{i}} \cdot \frac{\partial \vec{v}}{\partial x_{i}} d x
$$

and $(\vec{u}, \vec{v})$ the $\left[L^{2}(\Omega)\right]^{N}$ inner product $\int_{\Omega} \vec{u} \cdot \vec{v} d x$. We then seek a function $p \in$ $L^{2}(\Omega)$ such that

$$
(\operatorname{div} \vec{u}(p), q)=0 \quad \text { for all } q \in L^{2}(\Omega),
$$

where $\vec{u}(p)$ is the unique solution in $\left[H_{0}^{1}(\Omega)\right]^{N}$ of $a(\vec{u}(p), \vec{v})=(\vec{f}-\overrightarrow{\operatorname{grad}} p, \vec{v})$ for all $\vec{v} \in\left[H_{0}^{1}(\Omega)\right]^{N}$. It is easy to show that $(\vec{u}(p), p)$ solves Problem (P).

Received July 8, 1975.

AMS (MOS) subject classifications (1970). Primary 65N30.

* This work was supported by NSF grant MPS 74-05795. 
The approximation scheme is then

Problem $\left(\mathrm{P}_{h}\right)$ : Find $p_{h} \in T_{h_{2}}(\Omega)$ such that

$$
\left(\operatorname{div} \vec{u}_{h}\left(p_{h}\right), q_{h}\right)=0 \text { for all } q_{h} \in T_{h_{2}}(\Omega)
$$

where $\vec{u}_{h}\left(p_{h}\right)$ is the unique solution in $\left[T_{h_{1}}(\Omega)\right]^{N}$ of $a\left(\vec{u}_{h}\left(p_{h}\right), \vec{v}_{h}\right)=\left(\vec{f}-\overrightarrow{\operatorname{grad}} p_{h}\right.$, $\vec{v}_{h}$ ) for all $\vec{v}_{h} \in\left[T_{h_{1}}(\Omega)\right]^{N}$. $\left[T_{h_{1}}(\Omega)\right]^{N}$ and $T_{h_{2}}(\Omega)$ are finite dimensional subspaces of $\left[H_{0}^{1}(\Omega)\right]^{N}$ and $L^{2}(\Omega)$, respectively, and will be defined later.

We note that these equations can also be obtained by applying the method of Lagrange multipliers to the constraint $\operatorname{div} \vec{v}=0$.

In the next section we describe the notation and principal ideas to be used in the derivation of the error estimates.

2. Notation. Let $\Omega$ be a bounded domain in $\mathbf{R}^{N}$ with smooth boundary $\partial \Omega$. For $m$ a nonnegative integer, let $H^{m}(\Omega)$ denote the Sobolev space of order $m$ of functions defined on $\Omega$ with norm

$$
\|v\|_{m}=\left(\sum_{|\alpha| \leqslant m}\left\|D^{\alpha} v\right\|_{0}^{2}\right)^{1 / 2}, \text { where }\|v\|_{0}=\|v\|_{L^{2}(\Omega)} .
$$

Let $H_{0}^{1}(\Omega)$ denote the Sobolev space of functions in $H^{1}(\Omega)$ which "vanish" on $\partial \Omega$.

For vector-valued functions $\vec{v}=\left(v_{1}, \ldots, v_{N}\right)$ let $\left[H^{m}(\Omega)\right]^{N}$ be the space of $\vec{v}$ with components $v_{i} \in H^{m}(\Omega)$ and let

$$
\|\vec{v}\|_{m}=\left(\sum_{i=1}^{N}\left\|v_{i}\right\|_{m}^{2}\right)^{1 / 2}
$$

Finally, for convenience, define $\|\vec{v}\|_{E}^{2}=a(\vec{v}, \vec{v})$. We remark that $\|\vec{v}\|_{E}$ is a norm on $\left[H_{0}^{1}(\Omega)\right]^{N}$ equivalent with the $\|\vec{v}\|_{1}$ norm.

The following facts will be needed in the derivation of the error estimates.

Lemma 1 (SEe Teman [13]). Let $\Omega$ be an open set of class $C^{s}, s \geqslant 2$, and let $\vec{f} \in\left[H^{s-2}(\Omega)\right]^{N}$ and $g \in H^{s-1}(\Omega)$ be given with $\int_{\Omega} g d x=0$. Then there exist unique functions $\vec{u}$ and $p$ ( $p$ is unique up to a constant) which are solutions of the generalized Stokes problem

$$
\begin{aligned}
-v \Delta \vec{u}+\overrightarrow{\operatorname{grad} p} & =\vec{f} & & \text { in } \Omega, \\
\operatorname{div} \vec{u} & =g & & \text { in } \Omega, \\
\vec{u} & =\overrightarrow{0} & & \text { on } \partial \Omega,
\end{aligned}
$$

and satisfy $\vec{u} \in\left[H^{s}(\Omega)\right]^{N}, p \in H^{s-1}(\Omega)$, and the estimates

$$
\|\vec{u}\|_{s}+\|p\|_{s-1 / R} \leqslant C_{0}\left\{\|\vec{f}\|_{s-2}+\|g\|_{s-1}\right\}, \quad s \geqslant 1,
$$

where $C_{0}$ is a constant depending only on $\nu, s$, and $\Omega$.

$$
\left(\|p\|_{s-1 / R}=\inf _{c \in \mathbb{R}}\|p+c\|_{s-1}\right) \text {. }
$$


LEMMA 2 (E.G. SEE [12]). Let $\vec{w}(p)$ be the weak solution of the equation $-\nu \Delta \vec{w}(p)=-\overrightarrow{\operatorname{grad}} p$ with boundary condition $\vec{w}=\overrightarrow{0}$. Then if $\overrightarrow{\operatorname{grad} p} \in\left[L^{2}(\Omega)\right]^{N}$, $\vec{w}(p) \in\left[H^{2}(\Omega)\right]^{N}$ and $\|\vec{w}(p)\|_{2} \leqslant C\|\overrightarrow{\operatorname{grad}} p\|_{0}$, where $C$ is a constant independent of $p$.

LEMma 3. Let $\vec{w}(p)$ be the weak solution of the equation $-v \Delta \vec{w}(p)=$ $-\overrightarrow{\operatorname{grad}} p$ with boundary condition $\vec{w}(p)=\overrightarrow{0}$. If $p \in L^{2}(\Omega) / \mathbf{R}$, then $\vec{w}(p) \in\left[H_{0}^{1}(\Omega)\right]^{N}$ and $\|p\|_{0 / \mathbf{R}} \leqslant C_{1}\|\vec{w}(p)\|_{E} \leqslant C_{2}\|p\|_{0 / \mathbf{R}}$ for some constants $C_{1}, C_{2}$ independent of $p$.

Proof.

$$
\|p\|_{0 / \mathbf{R}}=\inf _{c \in \mathbf{R}}\|p+c\|_{0} \leqslant\|p-\bar{c}\|_{0}
$$

(where $\bar{c}=(\mu(\Omega))^{-1} \int_{\Omega} p(x) d x$ and $\mu(\Omega)$ denotes the measure of $\Omega$ ). Let $(z, \psi)$ be the solution of the generalized Stokes problem

$$
\begin{aligned}
-v \Delta \vec{z}+\overrightarrow{\operatorname{grad}} \psi & =0 & & \text { in } \Omega, \\
\operatorname{div} \vec{z} & =p-\bar{c} & & \text { in } \Omega, \\
\vec{z} & =0 & & \text { on } \partial \Omega .
\end{aligned}
$$

Then by Lemma $1, \vec{z}$ exists and is unique, and satisfies $\|\vec{z}\|_{E} \leqslant C\|p-\bar{c}\|_{0}$ for some constant $C$ independent of $p$. Hence

$$
\begin{aligned}
\|p-\bar{c}\|_{0}^{2} & \leqslant(p-\bar{c}, \operatorname{div} \vec{z})=(-\overrightarrow{\operatorname{grad} p}, \vec{z}) \\
& =a(\vec{w}(p), \vec{z}) \leqslant\|\vec{w}(p)\|_{E}\|\vec{z}\|_{E} \\
& \leqslant C\|\vec{w}(p)\|_{E}\|p-\bar{c}\|_{0} .
\end{aligned}
$$

Hence, $\|p\|_{0 / \mathrm{R}} \leqslant C\|\vec{w}(p)\|_{E}$. Also,

$$
\begin{aligned}
\|\vec{w}(p)\|_{E}^{2} & =\overrightarrow{(\operatorname{grad}} p, \vec{w}(p))=\overrightarrow{(\operatorname{grad}}[p+c], \vec{w}(p)) \quad(\text { for all constant } c) \\
& =(p+c, \operatorname{div} \vec{w}(p)) \leqslant\|p+c\|_{0}\|\operatorname{div} \vec{w}(p)\|_{0} \leqslant(\sqrt{N} / v)\|p+c\|_{0}\|\vec{w}(p)\|_{E} .
\end{aligned}
$$

Hence, $\|\vec{w}(p)\|_{E} \leqslant C\|p\|_{0 / \mathbf{R}}$.

We now introduce the finite dimensional subspaces we will be using in our approximation scheme. Following Babuška [4], we will define for all $0<h<1$ a one-parameter family of finite dimensional subspaces which we will denote $S_{h}^{t, k}(\Omega)$. We call $S_{h}^{t, k}(\Omega)$ a $(t, k)$-system for $t>k \geqslant 0$ if

(A1) $S_{h}^{t, k}(\Omega) \subset H^{k}(\Omega)$.

(A2) If $\varphi \in H^{l}(\Omega)$ and $0 \leqslant s \leqslant k \leqslant l$, then there exists $\phi_{h} \in S_{h}^{t, k}(\Omega)$ such that $\left\|\phi-\phi_{h}\right\|_{s} \leqslant C h^{\mu}\|\phi\|_{l}$, where $\mu=\min (l-s, t-s)$ and $C$ does not depend on $s, h$, or $\phi$. Note that the function $\phi_{h}$ may be different for different $s$.

If the function $\phi_{h}$ can be chosen independently of $s$, then the system will be called regular. We say that the regular system $S_{h}^{t, k}(\Omega)$ is strongly regular if its members satisfy

$$
\left\|\phi_{h}\right\|_{s} \leqslant C h^{-(s-m)}\left\|\phi_{h}\right\|_{m} \quad \text { for } 0 \leqslant m \leqslant s \leqslant k
$$

One final hypothesis that such systems may satisfy which we will require is

(A3) For $\phi \in H^{l}(\Omega) \cap H_{0}^{1}(\Omega)$, (A2) is satisfied by a $\phi_{h} \in S_{h}^{t, k}(\Omega) \cap H_{0}^{1}(\Omega)$. Systems satisfying these various hypotheses are constructed in [1] and [2]. 
We now proceed with the derivation of the error estimates.

\section{Error estimates.}

TheOrem 1. Suppose $\vec{f} \in\left[H^{r-2}(\Omega)\right]^{N}$ and $\vec{u}=\vec{u}(p)$ is the solution of Problem (P). Let $\vec{u}_{h}\left(p_{h}\right)$ be the solution of Problem $\left(\mathrm{P}_{h}\right)$ with $T_{h_{1}}(\Omega) a\left(t_{1}, k_{1}\right)$-system satisfying (A3) and $T_{h_{2}}(\Omega)$ a strongly $\left(t_{2}, k_{2}\right)$-regular system with $t_{1} \geqslant 2, k_{1} \geqslant 1$, and $k_{2} \geqslant 1$. If $h_{2} \geqslant K h_{1}$ for $K$ sufficiently large ( $K$ a constant independent of $\left.h_{1}\right)$, then there exists a constant $C$ independent of $h$ and $\vec{u}$ such that

$$
\left\|\vec{u}(p)-\vec{u}_{h}\left(p_{h}\right)\right\|_{1}+\left\|p-p_{h}\right\|_{L^{2} / \mathrm{R}} \leqslant C h^{\mu}\|\vec{f}\|_{r-2},
$$

where $h=\max \left(h_{1}, h_{2}\right)$ and $\mu=\min \left(r-1, t_{1}-1, t_{2}\right)$.

Proof. Since $\vec{u}(p)$ is the solution of Problem (P), (div $\vec{u}(p), q)=0$ for all $q \in L^{2}(\Omega)$. By (1), $\left(\operatorname{div} \vec{u}_{h}\left(p_{h}\right), q_{h}\right)=0$ for all $q_{h} \in T_{h_{2}}(\Omega)$. Hence $\left(\operatorname{div}\left[\vec{u}(p)-\vec{u}_{h}\left(p_{h}\right)\right], q_{h}\right)=0$ for all $q_{h} \in T_{h_{2}}(\Omega)$. Now

$$
\begin{aligned}
\left\|\vec{u}(p)-\vec{u}_{h}\left(p_{h}\right)\right\|_{E}^{2}= & a\left(\vec{u}(p)-\vec{u}_{h}\left(p_{h}\right), \vec{u}(p)-\vec{u}\left(q_{h}\right)\right) \\
& +a\left(\vec{u}(p)-\vec{u}_{h}\left(p_{h}\right), \vec{u}\left(q_{h}\right)-\vec{u}\left(p_{h}\right)\right) \\
& +a\left(\vec{u}(p)-\vec{u}_{h}\left(p_{h}\right), \vec{u}\left(p_{h}\right)-\vec{u}_{h}\left(p_{h}\right)\right) .
\end{aligned}
$$

We first observe that

$$
\begin{aligned}
a\left(\vec{u}(p)-\vec{u}_{h}\left(p_{h}\right), \vec{u}\left(q_{h}\right)-\vec{u}\left(p_{h}\right)\right) & \left.=\overrightarrow{(\operatorname{grad}}\left(p_{h}-q_{h}\right), \vec{u}(p)-\vec{u}_{h}\left(p_{h}\right)\right) \\
& =-\left(p_{h}-q_{h}, \operatorname{div}\left[\vec{u}(p)-\vec{u}_{h}\left(p_{h}\right)\right]\right)=0
\end{aligned}
$$

by the result obtained above. Applying the Schwarz inequality to the remaining two terms and collecting terms, we obtain

$$
1 / 2\left\|\vec{u}(p)-\vec{u}_{h}\left(p_{h}\right)\right\|_{E}^{2} \leqslant\left\|\vec{u}(p)-\vec{u}\left(q_{h}\right)\right\|_{E}^{2}+\left\|\vec{u}\left(p_{h}\right)-\vec{u}_{h}\left(p_{h}\right)\right\|_{E}^{2} .
$$

By Lemma $3,\left\|\vec{u}(p)-\vec{u}\left(q_{h}\right)\right\|_{E} \leqslant C\left\|p-q_{h}\right\|_{0}$. Hence, we obtain

$$
\left\|\vec{u}(p)-\vec{u}_{h}\left(p_{h}\right)\right\|_{E} \leqslant C\left[\left\|p-q_{h}\right\|_{0}+\left\|\vec{u}\left(p_{h}\right)-\vec{u}_{h}\left(p_{h}\right)\right\|_{E}\right] .
$$

Now by Lemma 3 ,

$$
\begin{aligned}
\left\|p-p_{h}\right\|_{0 / \mathrm{R}} & \leqslant C\left\|\vec{u}(p)-\vec{u}\left(p_{h}\right)\right\|_{E} \\
& \leqslant C\left[\left\|\vec{u}(p)-\vec{u}_{h}\left(p_{h}\right)\right\|_{E}+\left\|\vec{u}_{h}\left(p_{h}\right)-\vec{u}\left(p_{h}\right)\right\|_{E}\right] .
\end{aligned}
$$

Combining these results, we get

(2) $\left\|\vec{u}(p)-\vec{u}_{h}\left(p_{h}\right)\right\|_{E}+\left\|p-p_{h}\right\|_{0 / \mathbf{R}} \leqslant C\left[\left\|p-q_{h}\right\|_{0}+\left\|\vec{u}\left(p_{h}\right)-u_{h}\left(p_{h}\right)\right\|_{E}\right]$.

Using the approximation assumption (A2), the first term on the right of (2) is bounded by $C h_{2}^{\mu_{2}}$ where $\mu_{2}=\min \left(r-1, t_{2}\right)$. In order to estimate the second term we need to make use of the strong hypotheses we have made in the theorem about the approximation properties of the subspaces and their relationships. Now 
$\left\|\vec{u}\left(p_{h}\right)-\vec{u}_{h}\left(p_{h}\right)\right\|_{E} \leqslant\left\|\left[\vec{u}\left(p_{h}\right)-\vec{u}(p)\right]-\left[\vec{u}_{h}\left(p_{h}\right)-\vec{u}_{h}(p)\right]\right\|_{E}+\left\|\vec{u}(p)-\vec{u}_{h}(p)\right\|_{E}$.

Since

$$
a\left(\vec{u}(q)-\vec{u}_{h}(q), \vec{z}_{h}\right)=0 \text { for all } \vec{z}_{h} \in\left[T_{h_{1}}(\Omega)\right]^{N},
$$

we have for all $\vec{z}_{h}$ and $\vec{v}_{h} \in\left[T_{h_{1}}(\Omega)\right]^{N}$ that

$$
\begin{aligned}
\left\|\vec{u}\left(p_{h}\right)-\vec{u}_{h}\left(p_{h}\right)\right\|_{E} & \leqslant\left\|\vec{u}\left(p_{h}\right)-\vec{u}(p)-\vec{z}_{h}\right\|_{E}+\left\|\vec{u}(p)-\vec{v}_{h}\right\|_{E} \\
& \leqslant C h_{1}\left\|\vec{u}\left(p_{h}\right)-\vec{u}(p)\right\|_{2}+C h_{1}^{\mu}\|\vec{u}(p)\|_{r},
\end{aligned}
$$

where $\mu_{1}=\min \left(r-1, t_{1}-1\right)$ (by using (A2)).

Now

$$
\begin{aligned}
\left\|\vec{u}\left(p_{h}\right)-\vec{u}(p)\right\|_{2} & \leqslant C\left\|\overrightarrow{\operatorname{grad}}\left(p-p_{h}\right)\right\|_{0} \quad \text { (by Lemma 2), } \\
& \left.\leqslant C\left\|\overrightarrow{\operatorname{grad}}\left(p-p_{h}-c\right)\right\|_{0} \quad \text { (for all constant } c\right) \\
& \leqslant C\left\|p-p_{h}-c\right\|_{1} \leqslant C\left[\left\|p-q_{h}\right\|_{1}+\left\|q_{h}-p_{h}-c\right\|_{1}\right] \\
& \leqslant C\left[\left\|p-q_{h}\right\|_{1}+C h_{2}^{-1}\left\|q_{h}-p_{h}-c\right\|_{0}\right] \quad \text { (by strong regularity) } \\
& \leqslant C\left[\left\|p-q_{h}\right\|_{1}+C h_{2}^{-1}\left\|q_{h}-p\right\|_{0}+C h_{2}^{-1}\left\|p-p_{h}-c\right\|_{0}\right] .
\end{aligned}
$$

Applying the approximability assumption (A2), we obtain

$$
\begin{aligned}
\left\|\vec{u}\left(p_{h}\right)-\vec{u}_{h}\left(p_{h}\right)\right\|_{E} & \leqslant C h_{1}\left[h_{2}^{\mu} 2^{-1}\|p\|_{r-1}+C h_{2}^{-1}\left\|p-p_{h}-c\right\|_{0}\right]+C h_{1}^{\mu}\|\vec{u}(p)\|_{r} \\
& \leqslant C h_{1} h_{2}^{\mu_{2}-1}\|p\|_{r-1}+C K^{-1}\left\|p-p_{h}-c\right\|_{0}+C h_{1}^{\mu}\|\vec{u}(p)\|_{r} .
\end{aligned}
$$

Hence,

$$
\left\|\vec{u}\left(p_{h}\right)-\vec{u}_{h}\left(p_{h}\right)\right\|_{E} \leqslant C h_{1} h_{2}^{\mu_{2}-1}\|p\|_{r-1}+C K^{-1}\left\|p-p_{h}\right\|_{0 / R}+C h_{1}^{\mu_{1}}\|\vec{u}(p)\|_{r}
$$

(since the previous equation held for all constant $c$ ). Since $\|p\|_{r-1}$ and $\|\vec{u}(p)\|_{r}$ are bounded by $C\|\vec{f}\|_{r-2}$ by Lemma 1 , we have after collecting terms that for $K$ sufficiently large

$$
\left\|\vec{u}(p)-\vec{u}_{h}\left(p_{h}\right)\right\|_{E}+\left\|p-p_{h}\right\|_{0 / \mathrm{R}} \leqslant C\left[h_{2}^{\mu_{2}}+h_{1} h_{2}^{\mu_{2}-1}+h_{1}^{\mu_{1}}\right]\|\vec{f}\|_{r-2} .
$$

The theorem follows by setting $h=\max \left(h_{1}, h_{2}\right)$.

Theorem 2. Suppose $\vec{f} \in\left[H^{r-2}(\Omega)\right]^{N}$ and $\vec{u}=\vec{u}(p)$ is the solution of Problem (P). Let $\vec{u}_{h}\left(p_{h}\right)$ be the solution of Problem $\left(\mathrm{P}_{h}\right)$ with $T_{h_{1}}(\Omega) a\left(t_{1}, k_{1}\right)$-system satisfying (A3) and $T_{h_{2}}(\Omega) a\left(t_{2}, k_{2}\right)$-system with $k_{1} \geqslant 1, k_{2} \geqslant 0$. (Note that $T_{h_{2}}(\Omega)$ need not be strongly regular.) If there exists $\vec{v}_{h} \in\left[T_{h_{1}}(\Omega)\right]^{N}$ with $\left(\operatorname{div} \vec{v}_{h}, q_{h}\right)=0$ for all $q_{h} \in T_{h_{2}}(\Omega)$ such that

$$
\left\|\vec{u}-\vec{v}_{h}\right\|_{1} \leqslant C h_{1}^{\mu}\|\vec{u}(p)\|_{r}
$$

then 


$$
\left\|\vec{u}(p)-\vec{u}_{h}\left(p_{h}\right)\right\|_{1} \leqslant C h^{\mu}\|\vec{f}\|_{r-2},
$$

where $\mu=\min \left(r-1, \mu_{1}, t_{2}\right)$ and $h=\max \left(h_{1}, h_{2}\right)$.

Proof. Using the same argument as in Theorem 1, we have

$$
\begin{aligned}
\left\|\vec{u}(p)-\vec{u}_{h}\left(p_{h}\right)\right\|_{E}^{2}= & a\left(\vec{u}(p)-\vec{u}_{h}\left(p_{h}\right), \vec{u}(p)-\vec{u}\left(q_{h}\right)\right) \\
& +a\left(\vec{u}(p)-\vec{u}_{h}\left(p_{h}\right), \vec{u}\left(p_{h}\right)-\vec{u}_{h}\left(p_{h}\right)\right) .
\end{aligned}
$$

Since $a\left(\vec{z}_{h}, \vec{u}\left(p_{h}\right)-\vec{u}_{h}\left(p_{h}\right)\right)=0$ for all $\vec{z}_{h} \in\left[T_{h_{1}}(\Omega)\right]^{N}$, it follows that for all $\vec{v}_{h} \in$ $\left[T_{h_{1}}(\Omega)\right]^{N}$,

$$
\begin{aligned}
a(\vec{u}(p)- & \left.\vec{u}_{h}\left(p_{h}\right), \vec{u}\left(p_{h}\right)-\vec{u}_{h}\left(p_{h}\right)\right)=a\left(\vec{u}(p)-\vec{v}_{h}, \vec{u}\left(p_{h}\right)-\vec{u}_{h}\left(p_{h}\right)\right) \\
= & a\left(\vec{u}(p)-v_{h}, \vec{u}\left(p_{h}\right)-\vec{u}(p)\right)+a\left(\vec{u}(p)-\vec{v}_{h}, \vec{u}(p)-\vec{u}_{h}\left(p_{h}\right)\right) \\
= & \left(\vec{u}(p)-\vec{v}_{h}, \overrightarrow{\operatorname{grad}}\left(p-p_{h}\right)\right)+a\left(\vec{u}(p)-\vec{v}_{h}, \vec{u}(p)-\vec{u}_{h}\left(p_{h}\right)\right) \\
= & \left(\operatorname{div}\left[\vec{u}(p)-\vec{v}_{h}\right], p_{h}-p\right)+a\left(\vec{u}(p)-\vec{v}_{h}, \vec{u}(p)-\vec{u}_{h}\left(p_{h}\right)\right) \\
= & \left(\operatorname{div}\left[\vec{u}(p)-\vec{v}_{h}\right], p_{h}-q_{h}\right)+\left(\operatorname{div}\left[\vec{u}(p)-\vec{v}_{h}\right], q_{h}-p\right) \\
& +a\left(\vec{u}(p)-\vec{v}_{h}, \vec{u}(p)-\vec{u}_{h}\left(p_{h}\right)\right) .
\end{aligned}
$$

Since $\operatorname{div} \vec{u}(p)=0$ and $p_{h}-q_{h} \in T_{h_{2}}(\Omega)$, we have for all $\vec{v}_{h} \in\left[T_{h_{1}}(\Omega)\right]^{N}$ with (div $\vec{v}_{h}, q_{h}$ ) $=0$ for all $q_{h} \in T_{h_{2}}(\Omega)$ that

$$
\begin{aligned}
\left\|\vec{u}(p)-\vec{u}_{h}\left(p_{h}\right)\right\|_{E}^{2} \leqslant & a\left(\vec{u}(p)-\vec{u}_{h}\left(p_{h}\right), \vec{u}(p)-\vec{u}\left(q_{h}\right)\right) \\
& +\left(\operatorname{div}\left[\vec{u}(p)-\vec{v}_{h}\right], q_{h}-p\right)+a\left(\vec{u}(p)-\vec{v}_{h}, \vec{u}(p)-\vec{u}_{h}\left(p_{h}\right)\right) \\
\leqslant & \left\|\vec{u}(p)-\vec{u}_{h}\left(p_{h}\right)\right\|_{E}\left\|\vec{u}(p)-\vec{u}\left(q_{h}\right)\right\|_{E}+\left\|\operatorname{div}\left[\vec{u}(p)-\vec{v}_{h}\right]\right\|_{0}\left\|q_{h}-p\right\|_{0} \\
& +\left\|\vec{u}(p)-\vec{v}_{h}\right\|_{E}\left\|\vec{u}(p)-\vec{u}_{h}\left(p_{h}\right)\right\|_{E} .
\end{aligned}
$$

Applying the arithmetic-geometric mean inequality and Lemma 3 , we obtain

$$
\begin{aligned}
\left\|\vec{u}(p)-\vec{u}_{h}\left(p_{h}\right)\right\|_{E} & \leqslant C\left[\left\|\vec{u}(p)-\vec{v}_{h}\right\|_{1}+\left\|p-q_{h}\right\|_{0}\right] \\
& \leqslant C h_{2}^{\mu}{ }^{\mu}\|p\|_{r-1}+C h_{1}^{\mu}\|u(p)\|_{r}
\end{aligned}
$$

(by (A2) and the hypothesis of the theorem), where $\mu_{2}=\min \left(r-1, t_{2}\right)$. Hence, applying Lemma 1 , we obtain

$$
\left\|\vec{u}(p)-\vec{u}_{h}\left(p_{h}\right)\right\|_{E} \leqslant C h^{\mu}\|\vec{f}\|_{r-2},
$$

where $\mu=\min \left(r-1, \mu_{1}, t_{2}\right)$ and $h=\max \left(h_{1}, h_{2}\right)$.

Remark 1. One easy application of Theorem 2 occurs in the following case. Suppose $\Omega$ is a convex polygon in $\mathbf{R}^{2}$. Although the regularity result of Lemma 1 no longer applies, we know by a recent result of Kellogg and Osborn [10] that if $\vec{f} \in$ $\left[L^{2}(\Omega)\right]^{2}$, then $\vec{u}(p) \in\left[H^{2}(\Omega)\right]^{2}$ and $\|\vec{u}(p)\|_{2} \leqslant C\|\vec{f}\|_{0}$. Suppose we construct a triangulation $\Delta$ of $\Omega$ and define $T_{h_{1}}(\Omega)=\{v: v$ is continuous on $\Omega$, quadratic on each 
triangle $K$ of $\Delta$, and zero on $\partial \Omega$ \} and

$$
T_{h_{2}}(\Omega)=\{q: q \text { is constant on each triangle } K \text { of } \Delta\} .
$$

By a result in $[5]$, there exists an element $\vec{v}_{h} \in\left[T_{h_{1}}(\Omega)\right]^{2}$ such that

$$
\left(\operatorname{div} \vec{v}_{h}, q_{h}\right)=0 \quad \text { for all } q_{h} \in T_{h_{2}}(\Omega)
$$

and $\left\|\vec{u}(p)-\vec{v}_{h}\right\|_{1} \leqslant C h\|\vec{u}(p)\|_{2}$. Hence, by Theorem 2,

$$
\left\|\vec{u}(p)-\vec{u}_{h}\left(p_{h}\right)\right\|_{1} \leqslant C h\|\vec{f}\|_{0} .
$$

The key point is that not all the elements of $\left[T_{h_{1}}(\Omega)\right]^{2}$ have to satisfy (3). As long as one element does, we get the error estimate.

Remark 2. In the case described in Theorem 2 (i.e. without the inverse assumption and relation between the mesh sizes), it is no longer necessarily true that $p_{h}$ will be unique in $L^{2}(\Omega) / \mathbf{R}$. However, it is easy to verify that if there is any solution $p_{h}$ to Problem $\left(\mathrm{P}_{h}\right)$, then $\vec{u}_{h}\left(p_{h}\right)$ exists. Furthermore, if $p_{h}^{1}, p_{h}^{2}$ are two solutions of Problem $\left(\mathrm{P}_{h}\right)$, then $\vec{u}_{h}\left(p_{h}^{1}\right)=\vec{u}_{h}\left(p_{h}^{2}\right)$. We have

$$
\begin{aligned}
\left\|\vec{u}_{h}\left(p_{h}^{1}\right)-\vec{u}_{h}\left(p_{h}^{2}\right)\right\|_{E}^{2} & =\left(\vec{u}_{h}\left(p_{h}^{1}\right)-\vec{u}_{h}\left(p_{h}^{2}\right), \overrightarrow{\operatorname{grad}}\left(p_{h}^{2}-p_{h}^{1}\right)\right) \\
& =\left(\operatorname{div}\left[\vec{u}_{h}\left(p_{h}^{1}\right)-\vec{u}_{h}\left(p_{h}^{2}\right)\right], p_{h}^{1}-p_{h}^{2}\right)=0,
\end{aligned}
$$

since $\left(\operatorname{div} \vec{u}_{h}\left(p_{h}^{k}\right), q_{h}\right)=0$ for all $q_{h} \in T_{h_{2}}(\Omega), k=1,2$.

The above result also gives us uniqueness of $\vec{u}_{h}\left(p_{h}\right)$ in the case of Theorem 1 .

The existence and uniqueness of $p_{n}$ (in $L^{2}(\Omega) / \mathbf{R}$ ) are easily proved under the hypotheses of Theorem 1. By Lemma 3,

$$
\begin{aligned}
\left\|p_{h}^{1}-p_{h}^{2}\right\|_{0 / R} \leqslant & C\left\|\vec{u}\left(p_{h}^{1}\right)-\vec{u}\left(p_{h}^{2}\right)\right\|_{E} \\
= & C\left\|\vec{u}\left(p_{h}^{1}\right)-\vec{u}\left(p_{h}^{2}\right)-\left[\vec{u}_{h}\left(p_{h}^{1}\right)-\vec{u}_{h}\left(p_{h}^{2}\right)\right]\right\|_{E} \\
\leqslant & \leqslant\left\|\vec{u}\left(p_{h}^{1}\right)-\vec{u}\left(p_{h}^{2}\right)-\vec{z}_{h}\right\|_{E} \quad \text { for all } \vec{z}_{h} \in\left[T_{h_{1}}(\Omega)\right]^{N} \\
& \quad\left(\operatorname{since} a\left(\vec{u}(q)-\vec{u}_{h}(q), \vec{v}_{h}\right)=0 \quad \text { for all } \vec{v}_{h} \in\left[T_{h_{1}}(\Omega)\right]^{N}\right) \\
\leqslant & C C h_{1}\left\|\vec{u}\left(p_{h}^{1}\right)-\vec{u}\left(p_{h}^{2}\right)\right\|_{2} \quad(\text { by (A2)) } \\
\leqslant & C C h_{1}\left\|\overrightarrow{\operatorname{grad}}\left(p_{h}^{1}-p_{h}^{2}\right)\right\|_{0} \quad(\text { by Lemma 2) } \\
\leqslant & \left.\leqslant C h_{1}\left\|\overrightarrow{\operatorname{grad}}\left(p_{h}^{1}-p_{h}^{2}-c\right)\right\|_{0} \quad \text { (for all constant } c\right) \\
\leqslant & \left(C h_{1} / h_{2}\right)\left\|p_{h}^{1}-p_{h}^{2}-c\right\|_{0} \quad \text { (by strong regularity). }
\end{aligned}
$$

Hence,

$$
\left\|p_{h}^{1}-p_{h}^{2}\right\|_{0 / \mathrm{R}} \leqslant C K^{-1}\left\|p_{h}^{1}-p_{h}^{2}\right\|_{0 / \mathrm{R}}
$$

which implies that for $K$ sufficiently large $\left\|p_{h}^{1}-p_{h}^{2}\right\|_{0 / \mathrm{R}}=0$. 
By an extension of the duality argument we have the following estimates for the error in $\left[L^{2}(\Omega)\right]^{N}$.

THEOREM 3. Under the hypothesis of Theorem 1,

$$
\left\|\vec{u}(p)-\vec{u}_{h}\left(p_{h}\right)\right\|_{0} \leqslant C h^{\mu+1}\|\vec{f}\|_{r-2},
$$

where $\mu=\min \left(r-1, t_{1}-1, t_{2}\right)$ and $h=\max \left(h_{1}, h_{2}\right)$.

Proof. Let $(\vec{w}, Q)$ be the solution of

$$
\begin{aligned}
-v \Delta \vec{w}+\overrightarrow{\operatorname{grad}} Q & =\vec{u}(p)-\vec{u}_{h}\left(p_{h}\right) \text { in } \Omega, \\
\operatorname{div} \vec{w} & =0 \text { in } \Omega, \\
\vec{w} & =0 \text { on } \partial \Omega .
\end{aligned}
$$

Then,

$$
\begin{aligned}
\left\|\vec{u}(p)-\vec{u}_{h}\left(p_{h}\right)\right\|_{0}^{2} & =\left(\vec{u}(p)-\vec{u}_{h}\left(p_{h}\right),-\nu \Delta \vec{w}+\operatorname{grad} Q\right) \\
& =a\left(\vec{u}(p)-\vec{u}_{h}\left(p_{h}\right), \vec{w}\right)-\left(\operatorname{div}\left[\vec{u}(p)-\vec{u}_{h}\left(p_{h}\right)\right], Q\right) .
\end{aligned}
$$

Now by the definitions of $\vec{u}(p), \vec{u}_{h}\left(p_{h}\right)$ we have

$$
a\left(\vec{u}(p)-\vec{u}_{h}\left(p_{h}\right), \vec{v}_{h}\right)=\left(\overrightarrow{\operatorname{grad}} p_{h}-p, \vec{v}_{h}\right)=\left(p-p_{h}, \operatorname{div} \vec{v}_{h}\right)
$$

for all $\vec{v}_{h} \in\left[T_{h_{1}}(\Omega)\right]^{N}$, and $\left(\operatorname{div}\left[\vec{u}(p)-\vec{u}_{h}\left(p_{h}\right)\right], q_{h}\right)=0$ for all $q_{h} \in T_{h_{2}}(\Omega)$.

Subtracting, we obtain

$$
\begin{aligned}
& \left\|\vec{u}(p)-\vec{u}_{h}\left(p_{h}\right)\right\|_{0}^{2}=a\left(\vec{u}(p)-\vec{u}_{h}\left(p_{h}\right), \vec{w}-\vec{v}_{h}\right) \\
& \quad-\left(\operatorname{div}\left[\vec{u}(p)-\vec{u}_{h}\left(p_{h}\right)\right], Q-q_{h}\right)+\left(p-p_{h}, \operatorname{div}\left[\vec{v}_{h}-\vec{w}\right]\right) \\
& \leqslant \\
& \quad\left\|\vec{u}(p)-\vec{u}_{h}\left(p_{h}\right)\right\|_{E}\left\|\vec{w}-\vec{v}_{h}\right\|_{E}+\left\|\operatorname{div}\left[\vec{u}(p)-\vec{u}_{h}\left(p_{h}\right)\right]\right\|_{0}\left\|Q-q_{h}\right\|_{0} \\
& \quad+\left\|p-p_{h}\right\|_{0 / R}\left\|\operatorname{div}\left[\overrightarrow{v_{h}}-\vec{w}\right]\right\|_{0} \\
& \leqslant C h^{\mu}\|\vec{f}\|_{r-2} \cdot C h_{1}\|\vec{w}\|_{2}+C h^{\mu}\|\vec{f}\|_{r-2} C h_{2}\|Q\|_{1} \\
& \quad+C h^{\mu}\|\vec{f}\|_{r-2} C h_{1}\|\vec{w}\|_{2} \text { (by Theorem } 1 \text { and (A2)). }
\end{aligned}
$$

Since $\|\vec{w}\|_{2}+\|Q\|_{1} \leqslant C\left\|\vec{u}(p)-\vec{u}_{h}\left(p_{h}\right)\right\|_{0}$ by Lemma 1 , we have upon collecting terms that

$$
\left\|\vec{u}(p)-\vec{u}_{h}\left(p_{h}\right)\right\|_{0} \leqslant C h^{\mu+1}\|\vec{f}\|_{r-2},
$$

where $\mu=\min \left(r-1, t_{1}-1, t_{2}\right)$ and $h=\max \left(h_{1}, h_{2}\right)$.

\section{Department of Mathematics} Rutgers University

New Brunswick, New Jersey 08903

1. I. BABUŠKA, "Approximation by Hill functions," Comment. Math. Univ. Carolinae, v. 11, 1970, pp. 787-811. MR 45\#1396. 
2. I. BABUŠKA, Approximation by Hill Functions. II, Technical Note BN-708, Institute for Fluid Dynamics and Applied Mathematics, University of Maryland, 1971.

3. I. BABUŠKA, "The finite element method with Lagrangian multipliers," Numer. Math. v. 20, 1973, pp. 179-192.

4. I. BABUŠKA, The Mathematical Foundations of the Finite Element Method with Applications to Partial Differential Equtions, A. K. Aziz (editor), Academic Press, New York, 1972.

5. M. CROUSEIX \& P. RAVIART, Conforming and Nonconforming Finite Elenent Methods for Solving the Stationary Stokes Equations. I, Revue Française d'Automatique, Informatique et Recherche Operationelle, 7 année, decembre 1973, R-3, pp. 33-76.

6. R. S. FALK, "An analysis of the penalty method and extrapolation for the stationary Stokes equations," in Advances in Computer Methods for Partial Differential Equations,

R. Vichnevetsky (editor), Proceedings of the AICA Symposium, Lehigh Univ., June, 1975, pp. 66-69.

7. R. S. FALK \& J. T. KING, "A penalty and extrapolation method for the stationary Stokes equations, " SIAM J. Numer. Anal. (To appear.)

8. R. S. FALK, "A finite element method for the stationary Stokes equations using trial functions which do not have to satisfy div $\vec{v}=0$," Math. Comp. (To appear.)

9. R. S. FALK, A Ritz Method Based on a Complimentary Variational Principle, Revue Francaise d'Automatique, Informatique et Recherche Operationelle. (To appear.)

10. R. B. KELLOGG \& J. E. OSBORN, A Regularity Result for the Stokes Problem in a Convex Polygon, Technical Note BN-804, Institute for Fluid Dynamics and Applied Mathematics. University of Maryland, 1974.

11. O. A. LADY ŽENSKAJA, The Mathematical Theory of Viscous Incompressible Flow, Fizmatigiz, Moscow, 1961; English transl., Gordon and Breach, New York, 1962. MR 27 \#5034a, b.

12. J.- L. LIONS \& E. MAGENES, Problèmes aux limites non homogènes et applications, Vol. 1, Travaux et Recherches Mathématiques, no. 17, Dunod, Paris, 1968. MR 40 \#512.

13. R. TEMAM, On the Theory and Numerical Analysis of the Navier-Stokes Equations, Lecture Note \#9, University of Maryland, June, 1973. 